\title{
Invarianza Factorial del Multidimensional Body Self Relations Questionnaire (MBSRQ-M) en Hombres y Mujeres Universitarios Mexicanos
}

\author{
Iván Hinojosa, \\ Miguel Conchas Ramírez, \\ Humberto Blanco Vega, \\ Omar Ricardo Ortiz, \\ María del Carmen Zueck, \\ Jesús Enrique Peinado, \\ Juan de Dios Alvarado, \\ Yunuen Socorro Rangel Ledezma, \\ Facultad de Ciencias de la Cultura Física \\ Universidad Autónoma de Chihuahua, México
}

Doi:10.19044/esj.2020.v16n29p14 ～URL:http://dx.doi.org/10.19044/esj.2020.v16n29p14

\section{Resumen}

El presente estudio tiene como objetivo principal analizar las propiedades psicométricas versión española del Multidimensional Body Self Relations Questionnaire propuesta por Blanco, Viciana, Aguirre, Zueck, and Benavides (2017) en hombres y mujeres universitarios, para población mexicana. La muestra total fue de 1221 participantes; 653 mujeres y 568 hombres, con una edad media de 20.39 años $(\mathrm{DE}=1.83)$ y 20.76 años $(\mathrm{DE}=$ 1.95 ) respectivamente. De acuerdo a los requisitos psicométricos establecidos, los análisis factoriales confirmatorios mostraron que una estructura bifactorial (importancia subjetiva de la apariencia física e importancia subjetiva de la forma física) es viable $\mathrm{y}$ adecuada para ambas poblaciones (mujeres $\mathrm{y}$ hombres). Además, la estructura factorial, las cargas factoriales y los interceptos se consideran invariantes en las dos poblaciones; sin embargo, existen diferencias entre las poblaciones para las medias de los factores importancia subjetiva de la apariencia física e importancia subjetiva de la forma física.

Palabras clave: Estructura factorial, imagen corporal, sexo, validación de constructo 


\title{
Factorial Invariance of the Multidimensional Body Self Relations Questionnaire (MBSRQ-M) for Men and Women in Mexican University
}

\author{
Iván Hinojosa, \\ Miguel Conchas Ramírez, \\ Humberto Blanco Vega, \\ Omar Ricardo Ortiz, \\ María del Carmen Zueck, \\ Jesús Enrique Peinado, \\ Juan de Dios Alvarado, \\ Yunuen Socorro Rangel Ledezma, \\ Facultad de Ciencias de la Cultura Física \\ Universidad Autónoma de Chihuahua, México
}

\begin{abstract}
This paper focuses on analyzing the psychometric properties of the Spanish version of the Multidimensional Body Self Relations Questionnaire proposed by Blanco, Viciana, Aguirre, Zueck and Benavides (2017) in Mexican university men and women. The total sample consisted of 1221 participants (653 women and 568 men) with a mean age of 20.39 years $(\mathrm{SD}=$ $1.83)$ and 20.76 years $(S D=1.95)$, respectively. Based on the established psychometric requirements, confirmatory factor analysis show that a twofactor structure (subjective importance of physical appearance and subjective importance of physical form) is feasible and adequate for both populations (women and men). Furthermore, the factor structure, the factor loadings, and the intercepts are considered to be invariant in the two populations. There are differences between populations for the means of the factors, i.e., subjective importance of physical appearance and subjective importance of physical form.
\end{abstract}

Keywords: Factor structure, body image, sex, construct validation

\section{Introducción}

La imagen corporal es una idea multidimensional de la percepción del propio cuerpo, la cual consta de tres componentes principales: percepción, subjetividad y conductual (Velázquez, Vázquez, Mancilla, \& Ruíz, 2014), así 
como de las emociones que ésta genera (Cash, 2015); por ello, repercute en la salud física y mental, por ejemplo una imagen distorsionada o la presencia de insatisfacción corporal por presiones familiares, sociales que generan inseguridad en la persona, puede desencadenar estados de ánimos bajos (Raich, 2017).

Según lo anterior, un ejemplo son los adolescentes, Ya que estos son una población vulnerable relacionada con la insatisfacción corporal por los cambios experimentados en esta etapa, así como el estatus socioeconómico, nutricional y la calidad de la dieta, actividad física y a la condición física presentada (Castañeda, Zagalaz, Arufe, \& Campos-Mesa, 2018).

El hecho que la actividad física se encuentra de moda o que los familiares lo realicen, son condiciones con menor influencia entre los jóvenes. Es decir, influyen con mayor fuerza las motivaciones intrínsecas que extrínsecas (Castañeda et al., 2018; Santamaría, Cámara, \& Jiménez, 2019), otros motivos para realizar actividad física son para desarrollar competencias, la imagen o reconocimiento social. Sin embargo, una barrera constante, para la participación, es la incompatibilidad de los horarios de las actividades con las obligaciones académicas (Santamaría et al., 2019).

Sobre el género, los hombres manifiestan deseos de mayor volumen corporal, por otra parte las mujeres prefieren menos, ya que suelen percibirse con mayor peso corporal (Duno \& Acosta, 2019). Con ello, las motivaciones para realizar actividad para mejorar la salud también difieren: los hombres lo consideran una actividad lúdica, relaciones sociales y competición; en cambio las mujeres van dirigida a la salud y estética, es decir mantenerse en forma, mantener la salud, mejorar la imagen o el aspecto físico. Sin embargo, la falta de actividad física las respuestas van desde falta de disponibilidad temporal hasta de interés, pasando por cansancio, pereza y desgana, así como falta de gusto por la actividad, en ambos géneros.

Las mujeres suelen referirse más delgadas, debido a la cultura; los hombres prefieren una silueta mayor a la que realmente presentan (Sánchez, Suárez, \& Smith, 2018). El nivel socio económico también influye en la percepción de la figura de los jóvenes, de alto nivel manifiestan insatisfacción con su imagen y un constante deseo de perder peso mediante la realización de actividad física (Rodríguez, 2019).

La valoración y atención a la apariencia genera preocupación y acción hacia ésta misma y con ello una insatisfacción de la apariencia (Argyrides \& Kkeli, 2013). A pesar de la presencia de estas características, son pocos los universitarios que se registran con actividades físico-deportivas para cuidar la salud o desarrollar habilidades (Santamaría et al., 2019).

El uso de valoraciones de imagen corporal ayuda en problemas clínicos, fenómenos psicosociales, no necesariamente patológicos (Botella, Ribas, \& Ruiz, 2009), por ello la necesidad de generar o validar instrumentos 
relacionados con su medición. A través del tiempo se han generado encuestas de valoración con relación a las creencias y sentimientos del propio cuerpo, la cognición, importancia y actitud sobre el propio cuerpo, la cantidad de ítems ha ido variando con el tiempo, éstos se basan en la apariencia, su orientación, valoración, satisfacción, preocupación y determinación del peso (Cash, 2015).

El cuestionario Multidimensional Body Self Relations Questionnaire (MBSRQ) se ha analizado en varios idiomas como el alemán, griego, inglés, francés y español, con ello se reafirma como una evaluación multidimensional relacionado con la imagen corporal, con pequeñas variaciones en las subescalas, pero con ello se apoya a la generación de investigaciones de comparación entre diversas culturas (Vossbeck-Elsebusch et al., 2014). En personas con edad de 15 años o más (Cash, 2000), hasta los 46 años, en población considerada como no clínica, es decir que presenten trastornos de conducta alimentaria o de otro tipo (Roncero, Perpiñá, Marco, \& SánchezReales, 2015).

En España se ha recomendado un cuestionario más breve que el original (Botella et al., 2009); por otra parte, en México, se ha utilizado para conocer la percepción de hombres sobre la imagen corporal (Velázquez et al., 2014); así como en universitarios de ambos géneros (Blanco et al., 2017). Esta última aplicación presentó una consistencia interna adecuada y alta congruencia en los dos factores obtenidos, así como simple, viable y válida, a pesar de no coincidir con el modelo aplicado en España, ya que se tuvieron que eliminar ítems para lograr un mejor ajuste, atribuyéndose a diferencias sociales y culturales con los otros estudios

El presente estudio instrumental se ha dirigido a proporcionar apoyo empírico a la división factorial propuesta por Blanco et al. (2017) para la versión española del Multidimensional Body Self Relations Questionnaire (MBSRQ-M); lo que se justifica por la importancia de comprobar la estructura factorial de un instrumento y la equivalencia psicométrica del mismo en distintos grupos; ya que en el contexto de la comparación intergrupal, es indispensable plantearse la necesidad de llevar a cabo la adaptación de un instrumento de medida psicológica que cumpla con todos los criterios de equivalencia, pero sobre todo plantearse si la misma estructura factorial es aplicable a distintos grupos de sujetos o, de modo más genérico, a distintas poblaciones (Abalo, Lévy, Rial, \& Varela, 2006; Arbuckle, 2012).

Este trabajo pretende, por una parte, indagar si se replican los resultados psicométricos propuestos por L. H. Blanco et al. (2017) para el MBSRQ-M y, por otra, ampliarlos. Para ello, en primer lugar se comprobará el grado de congruencia de la estructura factorial del MBSRQ-M obtenida en el presente estudio y la reportada por Blanco et al. (2017). En segundo lugar, se calcula la invarianza factorial entre las muestras del presente estudio. Por 
ello la hipótesis consiste en el grado de congruencia de la estructura factorial de MBSRQ-M son similares para aportar mayor información sobre esta.

\section{Método}

\section{Participantes}

La muestra de 1221 participantes, 653 (53.5\%) mujeres y $568(46.5 \%)$ hombres, se obtuvo mediante un muestreo por conveniencia, abarcando la representatividad de las diferentes licenciaturas que se ofrecen en la Facultad de Ciencias de la Cultura Física la Universidad Autónoma de Chihuahua. Las edades de las mujeres fluctúan entre los 18 y 26 años, con una $\mathrm{M}=20.39$ ( $\mathrm{DE}=1.83)$; y las de los hombres fluctúan entre los 18 y 26 años, con una $\mathrm{M}=20.76(\mathrm{DE}=1.95)$.

\section{Instrumento}

La versión española reducida y adaptada del Multidimensional Body Self Relations Questionnaire (MBSRQ-M) de Blanco et al. (2017), para población mexicana, consta de 14 ítems que se agrupan en dos dimensiones: importancia subjetiva de la apariencia física ( 8 ítems) importancia subjetiva de la forma física (6 ítems). Se trata de una escala tipo Likert, asistida por computadora, de 5 puntos que va desde 0 "Totalmente en desacuerdo/Muy insatisfecho" a 5 "Totalmente de acuerdo/Muy satisfecho/a". De acuerdo a Blanco et al. (2017) el MBSRQ-M obtuvo buena consistencia interna y validez en todos sus factores.

\section{Procedimiento}

Se invitó a participar en el estudio a los alumnos de las licenciaturas que se ofrecen en la Facultad de Ciencias de la Cultura Física (FCCF) de la Universidad Autónoma de Chihuahua. Los que aceptaron participar firmaron la carta de aceptación correspondiente. Luego se aplicó el instrumento, antes descrito, por medio de una computadora personal (módulo administrador del instrumento del editor de escalas de ejecución típica), en una sesión de aproximadamente 25 minutos; en los laboratorios o centros de cómputo de la FCCF. Al inicio de cada sesión se hizo una pequeña introducción sobre la importancia de la investigación y de cómo acceder al instrumento. Se les solicitó la máxima sinceridad y se les garantizó la confidencialidad de los datos que se obtuvieran. Las instrucciones de cómo responder se encontraban en las primeras pantallas; antes del primer reactivo del instrumento. Al término de la sesión se les agradeció su participación. Una vez aplicado el instrumento se procedió a recopilar los resultados por medio del módulo generador de resultados del editor de escalas versión 2.0 (Blanco et al., 2013). 


\section{Análisis de datos}

El análisis psicométrico se realizó en dos etapas: 1) análisis factorial confirmatorio y 2) análisis de invarianza factorial; con el fin de obtener una prueba que presente las mejores propiedades para la conformación de los puntajes del MBSRQ-M.

Para conducir el análisis factorial confirmatorio para cada muestra, se utilizó el software AMOS 21 (Arbuckle, 2012), las varianzas de los términos de error fueron especificados como parámetros libres, en cada variable latente (factor) se fijó uno de los coeficientes estructurales asociados a uno, para que su escala sea igual a la de una de las variables superficiales (ítems). El método de estimación empleado fue el de Máxima Verosimilitud; siguiendo la recomendación de Thompson (2004), en el sentido de que cuando se emplea análisis factorial confirmatorio se debe corroborar no sólo el ajuste de un modelo teórico sino que es recomendable comparar los índices de ajuste de varios modelos alternativos para seleccionar el mejor.

Para evaluar el ajuste del modelo se emplearon el estadístico Chicuadrado, el índice de bondad de ajuste (GFI) y el error cuadrático medio de aproximación (RMSEA) como medidas absolutas de ajuste. El índice de bondad ajustado (AGFI), el Índice Tucker-Lewis (TLI) y el índice de bondad de ajuste comparativo (CFI) como medidas de ajuste incremental. El índice de ajuste normado de parsimonia (PNFI), el índice de calidad de ajuste de parsimonia (PGFI), la razón de Chi-cuadrado sobre los grados de libertad (CMIN/GL) y el Criterio de Información de Akaike (AIC) como medidas de ajuste de parsimonia (Gelabert et al., 2011).

Por último, se llevó a cabo un análisis de la invarianza factorial de los modelos de medida obtenidos, siguiendo las recomendaciones de Abalo et al. (2006), y se calculó la fiabilidad de cada una de las dimensiones a través del Alfa de Cronbach y el Coeficiente Omega (Revelle y Zinbarg, 2009). Todo lo anterior para sustentar y corroborar el anális realizado por Balnaco et al. (2017).

\section{Resultados}

\section{Análisis factoriales confirmatorios}

De acuerdo a los resultados de la Tabla 1 el análisis factorial confirmatorio de 14 ítems agrupados en dos factores en la muestra de mujeres es óptimo (GFI .959 y RMSEA .050) y de acuerdo a las medidas de ajuste incremental $y$ de parsimonia significativamente superior al modelo independiente y muy similar al modelo saturado.

Por otro lado, el análisis factorial confirmatorio en la muestra de hombres (Tabla 1), indica que el modelo de medición de dos factores es también óptimo (GFI .946 y RMSEA .059) y de acuerdo a las medidas de ajuste incremental y de parsimonia significativamente superior al modelo independiente y muy similar al modelo saturado. 
Tabla 1. Índices absolutos, incrementales y de parsimonia para los modelos generados. Análisis factorial confirmatorio para mujeres y hombres.

\begin{tabular}{|c|c|c|c|c|c|c|c|c|}
\hline \multirow[b]{2}{*}{ Modelo } & \multicolumn{3}{|c|}{ Índices absolutos } & \multicolumn{3}{|c|}{ Índices incrementales } & \multicolumn{2}{|c|}{ Índices de parsimonia } \\
\hline & $\chi^{2}$ & GFI & RMSEA & AGFI & TLI & CFI & CMIN/DF & AIC \\
\hline \multicolumn{9}{|c|}{ Solución factorial para as mujeres } \\
\hline 2 factores & $188.012 *$ & .959 & .050 & .940 & .967 & .974 & 2.648 & 256.012 \\
\hline Saturado & 0.000 & 1.000 & & & & 1.000 & & 210.000 \\
\hline Independiente & $4627.140 *$ & .319 & .277 & .215 & .000 & .000 & 50.848 & 4655.140 \\
\hline \multicolumn{9}{|c|}{ Solución factorial para los hombres } \\
\hline 2 factores & $213.328 *$ & .946 & .059 & .921 & .941 & .954 & 3.005 & 281.328 \\
\hline Saturado & 0.000 & 1.000 & & & & 1.000 & & 210.000 \\
\hline Independiente & $3187.294 *$ & .360 & .245 & .262 & .000 & .000 & 35.025 & 3215.294 \\
\hline
\end{tabular}

Nota: $* \mathrm{p}<.05 ;$ GFI $=$ índice de bondad de ajuste; RMSEA = raíz del error medio; AGFI = índice corregido de la bondad de ajuste; TLI = índice de Tucker-Lewis; $\mathrm{CFI}=$ índice de ajuste comparativo; $\mathrm{CMIN} / \mathrm{DF}=$ índice de ajuste chi cuadrado dividido por los grados de libertad; AIC = criterio de información de Akaike

De acuerdo a los resultados de la Tabla 2, ninguno de los 14 ítems en la muestra de mujeres y solo tres en la de hombres saturan por debajo de .60 en su dimensión prevista. Observándose además, intercorrelaciones moderadas entre los factores evidenciando una adecuada validez discriminante entre ellos.

Tabla 2. Soluciones estandarizadas análisis factorial confirmatorio en ambas muestras.

\begin{tabular}{|c|c|c|c|c|}
\hline \multirow[b]{2}{*}{ Item } & \multicolumn{2}{|c|}{ Mujeres } & \multicolumn{2}{|c|}{ Hombres } \\
\hline & F1 & $\mathrm{F} 2$ & $\overline{\mathrm{F} 1}$ & F2 \\
\hline \multicolumn{5}{|l|}{ Pesos Factoriales } \\
\hline 1 Mi cuerpo es atractivo & .74 & & .56 & \\
\hline $3 \mathrm{Me}$ gusta mi aspecto tal y como es & .77 & & .62 & \\
\hline 6 Me gusta el aspecto de mi cuerpo sin ropa & .81 & & .66 & \\
\hline $8 \mathrm{Me}$ gusta cómo me sienta la ropa & .72 & & .63 & \\
\hline 11 Grado de satisfacción con la parte media de tu cuerpo & .72 & & .67 & \\
\hline 12 Grado de satisfacción con tu tono muscular & .61 & & .66 & \\
\hline 13 Grado de satisfacción con tu peso corporal & .71 & & .74 & \\
\hline 14 Grado de satisfacción con tu aspecto general & .79 & & .82 & \\
\hline 2 No hago ejercicio regularmente & & .64 & & .54 \\
\hline $\begin{array}{l}4 \text { No realizo actividades que me mantengan en buena forma } \\
\text { física }\end{array}$ & & .71 & & .53 \\
\hline 5 Hago cosas que aumenten mi fuerza física & & .68 & & .60 \\
\hline 7 Me esfuerzo en mejorar mi resistencia física & & .70 & & .69 \\
\hline 9 Trato de estar físicamente activo & & .75 & & .81 \\
\hline 10 Hago deporte regularmente a lo largo del año & & .75 & & .70 \\
\hline \multicolumn{5}{|l|}{ Correlaciones Factoriales } \\
\hline F1 & - & .42 & - & .58 \\
\hline F2 &. .42 & - & .58 & - \\
\hline
\end{tabular}

Nota: $\mathrm{F} 1$ = Importancia subjetiva de la apariencia física, F2 = Importancia subjetiva de la forma física

\section{Invarianza de la estructura factorial entre mujeres y hombres universitarios}

Los índices de ajuste obtenidos (Tabla 3) permiten aceptar la equivalencia de los modelos de medida básicos entre las dos muestras. Aunque 
el valor de Chi-cuadrado excede al exigido para aceptar la hipótesis de invarianza, los índices GFI=.953, CFI=.966, RMSEA=.039 y AIC=537.346 contradicen esta conclusión lo que nos permite aceptar el modelo base de la invarianza (modelo sin restricciones).

Añadiendo al modelo base restricciones sobre las cargas factoriales caracterizamos la invarianza métrica. Los valores que se recogen en la Tabla 3 permiten aceptar este nivel de invarianza. El índice de ajuste general (GFI .947) y el error cuadrático medio de aproximación (RMSEA .040) siguen aportando información convergente en esta dirección. Además, el criterio de información de Akaike (AIC 565.369) y el índice comparativo de Bentler (CFI .961) no sufren grandes variaciones respecto al modelo anterior. Haciendo uso del criterio para la evaluación de los modelos anidados propuesto por Cheung and Rensvold (2002), quiénes sugieren que si el cálculo de la diferencia de los CFI de ambos modelos anidados disminuye en .01 o menos, se da por bueno el modelo restringido y por tanto el cumplimiento de la invarianza factorial; la diferencia entre CFIs obtenida permite aceptar el modelo de invarianza métrica. Podemos concluir hasta ahora que las cargas factoriales son equivalentes en las dos muestras.

Una vez demostrada la invarianza métrica entre las muestras, pasamos a evaluar la equivalencia entre interceptos (invarianza factorial fuerte). Los índices (Tabla 3) muestran un ajuste aceptable de este modelo, tanto evaluado de modo independiente como analizándolo respecto a su anidamiento con el modelo de invarianza métrica. La diferencia entre los índices comparativos de Bentler es nueve milésimos; el índice de ajuste general es .940 y el error cuadrático medio de aproximación es .044. Aceptada la invarianza fuerte, los dos modelos evaluados son equivalentes respecto a los coeficientes factoriales y a los interceptos.

Tabla 3. Índices de bondad de ajuste de cada uno de los modelos puestos a prueba en la invarianza factorial.

\begin{tabular}{llllllll}
\hline Modelo & \multicolumn{7}{l}{ Índice de Ajuste } \\
\cline { 2 - 7 } & $\chi^{2}$ & $\mathrm{gl}$ & GFI & PNFI & CFI & RMSEA AIC \\
\hline Modelo sin & $401.346^{*}$ & 142 & .953 & .740 & .966 & .039 & 537.346 \\
$\begin{array}{l}\text { restricciones } \\
\text { Invarianza métrica }\end{array}$ & $453.369^{*}$ & 154 & .947 & .797 & .961 & .040 & 565.369 \\
$\begin{array}{l}\text { Invarianza factorial } \\
\text { fuerte }\end{array}$ & $520.531^{*}$ & 157 & .940 & .805 & .952 & .044 & 626.531 \\
\hline
\end{tabular}

Nota: $* \mathrm{p}<.05 ; \mathrm{GFI}=$ índice de bondad de ajuste; PNFI = índice de ajuste normado de parsimonia; CFI = índice de ajuste comparativo; RMSEA = raíz del error medio; $\mathrm{AIC}=$ criterio de Información de Akaike

Los factores obtenidos en los análisis factoriales confirmatorios alcanzan, en su mayoría valores de consistencia interna por encima de .80 en ambas muestras (mujeres y hombres); evidenciando una consistencia interna 
adecuada para este tipo de subescalas, particularmente si se considera el número reducido de ítems (Tabla 4).

Tabla 4. Coeficiente omega y alfa para los factores obtenidos.

\begin{tabular}{|c|c|c|c|c|}
\hline \multirow[b]{2}{*}{ Factor } & \multicolumn{2}{|c|}{ Mujeres } & \multicolumn{2}{|c|}{ Hombres } \\
\hline & $\Omega$ & $\alpha$ & $\Omega$ & $\alpha$ \\
\hline $\begin{array}{l}\text { Importancia subjetiva de la } \\
\text { apariencia física }\end{array}$ & .904 & .903 & .868 & .867 \\
\hline $\begin{array}{l}\text { Importancia subjetiva de la } \\
\text { forma física }\end{array}$ & .856 & .861 & .813 & .802 \\
\hline
\end{tabular}

\section{Contrastes de las medias de los factores entre mujeres y hombres}

Una vez comprobada la invarianza factorial, las diferencias entre las medias de los factores de los dos grupos se estimaron tomando como referente la muestra de hombres, fijando en 0 el valor de las medias para dicha muestra y estimando libremente el valor de las medias para la muestra de mujeres. Las restricciones sobre los coeficientes de regresión e interceptos, requeridos para los contrastes entre las medias se realizaron automáticamente mediante el software AMOS 21 (Arbuckle, 2012). Los resultados de las comparaciones entre medias indicaron que las mujeres presentan, en ambos factores un mayor nivel de inconformidad con su imagen corporal: Importancia subjetiva de la apariencia física $(-.347, \mathrm{p}<0.001)$ e Importancia subjetiva de la forma física $(-.770, \mathrm{p}<0.001)$.

\section{Conclusiones}

De los resultados mostrados, de su análisis y de su discusión, y tomando en cuenta que el objetivo principal de este estudio fue el de examinar la estructura factorial de la versión española reducida y adaptada del Multidimensional Body Self Relations Questionnaire (MBSRQ-M) de Blanco et al. (2017), la hipótesis a trabajar fue relacionada con el grado de congruencia de la estructura factorial de MBSRQ-M son similares para aportar mayor información sobre esta y la medición de la invariancia de dicha estructura en alumnas y alumnos universitarios, se pueden obtener las siguientes conclusiones:

1) El Análisis Factorial Confirmatorio, en ambas muestras, indicó que el ajuste de los datos al modelo teórico de 14 ítems agrupados en dos factores es óptimo; resultados que concuerdan con los reportados por Blanco et al. (2017). Al mismo tiempo que los dos factores así obtenidos presentan en general saturaciones factoriales estandarizadas adecuadas. Por su parte los factores correlacionan entre sí de forma positiva y estadísticamente significativa lo cual muestra que a medida que aumenta nivel de inconformidad con la imagen corporal en alguno de los factores, también aumenta en el otro. 
2) Los dos factores obtenidos muestran una consistencia interna adecuada.

3) Conjuntamente con todo lo antes dicho, los resultados del análisis de la invarianza factorial entre las muestras; indican una alta congruencia entre pares de factores. Lo que sugiere la existencia de fuertes evidencias de la validación cruzada de la medida y por tanto de la estabilidad de la estructura, hasta que no se demuestre lo contrario.

4) Las comparaciones entre los grupos reflejaron diferencias significativas, a favor de los hombres en ambos factores (importancia subjetiva de la apariencia física e importancia subjetiva de la forma física). Lo que parece indicar que las mujeres se perciben con mayor ansiedad en lo que se refiere a su imagen corporal, lo que concuerda con lo reportado en otras investigaciones donde se afirma que las mujeres, en comparación con hombres de la misma edad y nivel de estudios, frecuentemente desean ser más delgadas o perder peso, independientemente de que sea necesario (Acosta, Llopis, GómezPeresmitré, y Pineda, 2005; Muñoz, Zueck, Blanco, Chávez, y Jasso, 2014) y se muestran más insatisfechas con su imagen corporal (Blanco et al., 2017; Mercado, 2008; Muñoz et al., 2014).

En síntesis, el análisis de las propiedades psicométricas, ha mostrado que una estructura bifactorial es viable y apropiada de acuerdo a los requisitos psicométricos establecidos cuando los informantes son los propias personas. La estructura de dos factores, atendiendo a criterios estadísticos y sustantivos, ha mostrado adecuados indicadores de ajuste, de fiabilidad y de validez. Sin embargo, consideramos que más estudios son necesarios con el fin de corroborar o refutar los datos obtenidos en la presente investigación.

\section{References:}

1. Abalo, J., Lévy, J., Rial, A., \& Varela, J. (2006). Invarianza factorial con muestras múltiples. In J. Lévy (Ed.), Modelización con Estructuras de Covarianzas en Ciencias Sociales (pp. 259-278). Netbiblo.

2. Acosta, M. V., Llopis, J. M., Gómez-Peresmitré, G., \& Pineda, G. (2005). Evaluación de la conducta alimentaria de riesgo. Estudio transcultural entre dolescentes de España y México. International Journal of Psychology and Psychological Therapy, 5(3), 223-232.

3. Arbuckle, J. R. (2012). AMOS users guide version 21.0. Chicago, IL: Marketing Department, SPSS Incorporated.

4. Argyrides, M. \& Kkeli, N. (2013). Multidimensional Body-Self Relations Questionnaire-Appearance Scales: Psychometric Properties 
of the Greek Version. Psychological Reports, 113(3), 885-897. doi: 10.2466/03.07.pr0.113x29z6

5. Blanco, H., Ornelas, M., Tristán, J. L., Cocca, A., Mayorga-Vega, D., López-Walle, J., \& Viciana, J. (2013). Editor for creating and applying computerise surveys. Procedia Social and Behavioral Sciences, 106, 935-940. doi: http://dx.doi.org/10.1016/j.sbspro.2013.12.105

6. Blanco, L. H., Viciana, J., Aguirre, J. F., Zueck, M. C., \& Benavides, E. V. (2017). Composición factorial del Multidimensional Body Self Relations Questionnaire (MBSRQ) en universitarios mexicanos. Revista de Psicología del Deporte, 26(2), 113-118.

7. Botella, L., Ribas, E., \& Ruiz, J. (2009). Evaluación Psicométrica de la Imagen Corporal: Validación de la versión española del multidimensional body self relations questionnaire (MBSRQ). Revista Argentina de Clínica Psicológica, 18(3), 253-264.

8. Cash, T. (2000). The MBSRQ users' manual (3 ed.).

9. Cash, T. (2015). Multidimensional Body-Self Relations Questionnaire (MBSRQ). Encyclopedia of Feeding and Eating Disorders, 1-4. doi: 10.1007/978-981-287-087-2_3-1

10. Castañeda, C., Zagalaz, M. L., Arufe, V., \& Campos-Mesa, M. C. (2018). Motivos hacia la práctica de actividad física de los estudiantes universitarios sevillanos. Revista Iberoamericana de Psicología del Ejercicio y el Deporte, 13(1), 79-89.

11. Cheung, G. W. \& Rensvold, R. B. (2002). Evaluating goodness-of-fit indexes for testing measurement invariance. Structural Equation Modeling, 9(2), 233-255. doi: 10.1207/s15328007SEM0902_5

12. Duno, M. \& Acosta, E. (2019). Percepción d ela imagen corporal en adolescentes universitarios. Revista chilena de nutrición, 46(5), 545553.

13. Gelabert, E., García-Esteve, L., Martín-Santos, R., Gutiérrez, F., Torres, A., \& Subirà, S. (2011). Psychometric properties of the Spanish version of the Frost Multidimensional Perfectionism Scale in women. Psicothema, 23(1), 133-139.

14. Mercado, C. (2008). Aplicación del análisis de género a los trastornos de la conducta alimentaria. Revista de Salud, 4(14), 1-14.

15. Muñoz, F., Zueck, M. C., Blanco, J. R., Chávez, A., \& Jasso, J. (2014). Body image perception of Mexican youth: A gender comparison. Education Journal, 3(5), 261-265. doi: 10.11648/j.edu.20140305.11

16. Raich, R. M. (2017). La tiranía del cuerpo ¿Por qué no me veo como soy? : Siglantana.

17. Revelle, W. \& Zinbarg, R. E. (2009). Coefficients alpha, beta, omega and the glb: comments on Sijtsma. Psychometrika, 74(1), 145-154. doi: 10.1007/s11336-008-9102-z 
18. Rodríguez, C. (2019). Imagen corporal y su relación con el nivel de actividad física y la inteligencia emocional en adolescentes femeninas de diferentes niveles socioeconómicos: estudio en un población argentina. (Doctoral dissertation), Universidad de Huelva.

19. Roncero, M., Perpiñá, C., Marco, J., \& Sánchez-Reales, S. (2015). Confirmatory factor analysis and psychometric properties of the Spanish version of the Multidimensional Body-Self Relations Questionnaire-Appearance Scales. Body Image, 14, 47-53. doi: 10.1016/j.bodyim.2015.03.005

20. Sánchez, G. F., Suárez, A. D., \& Smith, L. (2018). Analysis of body image and obesity by Stunkard's silhouettes in 3-to 18-year-old Spanish children and adolescents. Anales de psicología, 34(1), 167172.

21. Santamaría, M. C., Cámara, M. C., \& Jiménez, A. (2019). Diferencias en los motivos hacia la actividad física de los universitarios según nivel de actividad y su relación con la satisfacciónd el servicio deportivo universitario. Retos: nuevas tendencias en educación física, deporte y recreación (35), 191-195.

22. Thompson, B. (2004). Exploratory and Confirmatory Factor Analysis. Understanding concepts and applications. American Psychological Association.

23. Velázquez, H. J., Vázquez, R., Mancilla, J. M., \& Ruíz, A. O. (2014). Propiedades psicométricas del Multidimensional Body Self Relations Questionnaire (MBSRQ). Revista mexicana de trastornos alimentarios, 5(2), 107-114.

24. Vossbeck-Elsebusch, A. N., Waldorf, M., Legenbauer, T., Bauer, A., Cordes, M., \& Vocks, S. (2014). German version of the Multidimensional Body-Self Relations Questionnaire - Appearance Scales (MBSRQ-AS): Confirmatory factor analysis and validation. Body Image, 11(3), 191-200. doi: 10.1016/j.bodyim.2014.02.002 